\title{
MÉTODO OPTIMIZADO PARA EVALUAR ÁCIDOS BILIARES DE MUESTRAS FECALES SECAS O PRESERVADAS EN ETANOL COMO HERRAMIENTA PARA IDENTIFICAR CARNÍVOROS SILVESTRES
}

\author{
ArTuro SALAME-MÉNDEZ, ${ }^{1 *}$ MoISÉs ANDRADE-HERRERA, ${ }^{2}$ LEO \\ ZAMORA-TORRES, ${ }^{1}$ HÉCTOR SERRANO, ${ }^{3}$ SAÚL SOTO-MENDOZA, ${ }^{4}$ \\ ALONDRA CASTRO-CAMPILLO, ${ }^{2}$ JOSÉ RAMÍREZ-PULIDO $^{2}$ \& JORGE \\ HARO-CASTELLANOS ${ }^{1}$
}

Departamentos de Biología de la Reproducción ${ }^{1}$, Biología ${ }^{2}$, y Ciencias de la Salud ${ }^{3}$. División de Ciencias Biológicas y de la Salud. Universidad Autónoma Metropolitana, Unidad Iztapalapa. Av San Rafael Atlixco \# 186. Col. Vicentina. Iztapalapa, CP 09340. Apdo. Postal 55-535. México D. F. MÉXICO.

Área de Reproducción ${ }^{4}$. Dirección General de Zoológicos y Vida Silvestre. Zoológico de Chapultepec. Av. Chivatito S/N. San Miguel Chapultepec, México D. F. MÉXICO.

( ${ }^{*}$ Correspondencia: <asam@xanum.uam.mx>).

Salame-Méndez, A., M. Andrade-Herrera, L. Zamora-Torres, H. Serrano, S. Soto-Mendoza, A. Castro-Campillo, J. Ramírez-Pulido \& J. Haro-Castellanos. 2012. Método optimizado para evaluar ácidos biliares de muestras fecales secas o preservadas en etanol como herramienta para identificar carnívoros silvestres. Acta Zoológica Mexicana (n. s.), 28(2): 305-320.

RESUMEN. Se optimizan varios aspectos involucrados en el método para una obtención confiable, más sencilla y expedita de ácidos biliares (AB) y colesterol (Co), a partir de excretas de carnívoros silvestres, incluyendo: la selección de un revelador óptimo; la adecuación de la fase móvil en una primera evaluación cromatográfica; la optimización de la separación, mediante una segunda evaluación cromatográfica con su propia fase móvil; el uso de cantidades mínimas de muestra fecal (mg); así como de la solución etanólica $(\mu \mathrm{L})$ en donde fueron preservadas como materia prima. El nuevo método es más sencillo y fácilmente reproducible, por lo que resulta en una herramienta analítica indirecta, no-invasiva y sumamente viable para la identificación de carnívoros silvestres, a partir de los AB en sus excretas. Para probar el método se usaron excretas de Puma concolor, Panthera onca, Canis lupus baileyi y Canis latrans mantenidos en cautiverio, las cuales se describen, por lo que adicionalmente, en este trabajo se presenta por primera vez el perfil de $\mathrm{AB}$ en el lobo mexicano.

Palabras clave: Carnívoros, ácidos biliares, heces fecales, cromatografía en capa fina, identificación, métodos no-invasivos, Puma, Panthera, Canis.

Salame-Méndez, A., M. Andrade-Herrera, L. Zamora-Torres, H. Serrano, S. Soto-Mendoza, A. Castro-Campillo, J. Ramírez-Pulido \& J. Haro-Castellanos. 2012. Optimized method for

Recibido: 11/04/2011; aceptado: 06/02/2012. 
evaluation of bile acids from dry or ethanol preserved fecal samples as a tool for the identification of wild carnivores. Acta Zoológica Mexicana (n. s.), 28(2): 305-320.

ABSTRACT. We optimize several aspects involved in the method for a reliable, more simple, and easy obtaining of bile acids (AB) and cholesterol (Co) from the feces of wild carnivores, including: selection of the optimal developer; adaptation of the mobile phase in a first chromatographic evaluation; optimization of separation through a second chromatographic evaluation with another mobile phase; the use of minimal amounts of both fecal sample $(\mathrm{mg})$ or the ethanolic solution $(\mu \mathrm{L})$ in which the former were preserved. The new procedure is simpler and easily reproducible, and thus it turns out to be an analytic, non-invasive, and feasible tool for the identification of wild carnivores by means of the AB within their feces. In order to probe the method, we analyzed feces from Puma concolor, Panthera onca, Canis lupus baileyi, and Canis latrans maintained in captivity, which are also described and, therefore, we also present for the first time, the profile of $\mathrm{AB}$ for the Mexican wolf.

Keywords: Carnivores, bile acids, feces, thin layer chromatography, identification, non-invasive methods, Puma, Panthera, Canis.

\section{INTRODUCCIÓN}

Un problema a resolver cuando se utilizan heces fecales en estudios sobre la dinámica poblacional o la dieta de carnívoros en vida libre, es asegurarse de que la muestra pertenezca a la especie de interés y no a otra(s) especie(s) sintópica(s). Aún cuando la identificación de las heces a través de los sentidos sea hecha por un experto, se puede errar en la determinación de la especie, ya sea porque la apariencia, la textura, el olor o el tamaño de las heces son similares entre las distintas especies, lo cual es especialmente cierto si sólo se cuenta con muestras incompletas (Johnson et al. 1984, Cazón et al. 2009). Esta subjetividad en la asignación organoléptica de cierta muestra fecal a determinada especie, ha hecho necesario auxiliarse de métodos analíticos que aumenten la certidumbre de la identificación. Tal es el caso de la determinación del perfil de ácidos biliares $(\mathrm{AB})$ en las excretas por medio de cromatografía en capa fina (CCF o TLC por su acrónimo en inglés). Los primeros en aplicar y describir el procedimiento de la CCF para identificar carnívoros silvestres en vida libre, a partir de los AB contenidos en sus excretas, fueron Major et al. (1980) con base en el hallazgo de Haslewood (1967), quien encontró que había diferencias, tanto cualitativas como cuantitativas, entre los AB de diferentes especies de vertebrados. Más adelante, Quinn \& Jackman (1994) criticaron la propuesta de Major et al. (1980) al encontrar que el perfil de los $\mathrm{AB}$ cambiaba dentro de una misma especie, dependiendo de sus hábitos alimenticios (v. gr. los perfiles respectivos variaron entre animales alimentados en cautiverio, contra animales en vida libre).

No obstante este tipo de crítica o de otros factores que pueden afectar el perfil de los $\mathrm{AB}$, tales como la edad y el sexo de los individuos, el análisis de $\mathrm{AB}$ en las excretas ha sido principalmente utilizado para asegurar la identificación de especies medianas y grandes de mamíferos, naturalmente evasivos, en estudios sobre sus hábitos alimenticios, sus relaciones tróficas, su dinámica poblacional o para precisar, por ejemplo, qué especie de depredador ataca al ganado (Fernández et al. 1997, Taber et 
al. 1997, Ray \& Sunquist 2001, Amín et al. 2006, Guerrero et al. 2006, Cazón et al. 2009, García-Esponda et al. 2009). Sin embargo, cabe destacar que el perfil de los $\mathrm{AB}$ presentes en las excretas de carnívoros silvestres no ha sido más ampliamente utilizado, a pesar de su evidente utilidad, tanto en la identificación de carnívoros como en otras posibles aplicaciones prácticas para conocer su biología y ecología, aún cuando se trata de un enfoque no-invasivo y especialmente atractivo para abordar este tipo de especies que son naturalmente evasivas y que además suelen encontrarse en alguna categoría de riesgo para su conservación (NOM-059-SEMARNAT 2010).

Cuando nosotros revisamos las indicaciones para el uso de esta técnica que aparecían en la literatura, observamos: a) que los procedimientos descritos para la extracción de AB y Co, así como para realizar la CCF eran sumamente complejos y tardados, no obstante los esfuerzos por hacerlos más accesibles (Capurro et al. 1997, Fernández et al. 1997, Cazón \& Sühring 1999, Guerrero et al. 2006); b) que se requería de una inversión considerable al incluir equipo costoso y hasta innecesario $(v$. gr., rotavapor usado en la extracción), pero más importante aún, c) que las cantidades de muestra requeridas (i. e., 1 gr de muestra fresca o 3 gr de muestra seca sensu Johnson et al. 1984, Quinn \& Jackman 1994, Cazón \& Sühring 1999, Guerrero et al. 2006, Cazón et al. 2009) podrían comprometer el uso de una misma muestra fecal para diferentes propósitos ( $v$. gr., identificación por AB más valoración de hormonas esteroides sexuales), o bien, que no podrían obtenerse de otro tipo de especies como serían los micromamíferos.

Es por ello que el objetivo del presente trabajo fue optimizar varios de los aspectos del método para que los profesionales interesados en carnívoros silvestres, tuviesen a su alcance una herramienta más accesible para la identificación de especies en vida libre y que aún pudiesen usar las excretas recolectadas en el campo para otros fines. Para lograrlo abordamos varios de los aspectos y procedimientos involucrados con: a) la naturaleza y cantidad de materia prima para extraer los nueve $A B$ de referencia que han sido usados en otros estudios; b) con la extracción misma de los AB y c) con su separación y evaluación por medio de CCF. Al principio sólo usamos los nueve AB de referencia, pero después probamos el método resultante con muestras fecales de felinos (puma, Puma concolor; jaguar, Panthera onca) y de cánidos (lobo mexicano, Canis lupus baileyi; coyote, Canis latrans) silvestres, mantenidos en cautiverio. De esta manera, pudimos contar con excretas que podían ser referidas a determinada especie con certeza para probar nuestro método y para compararlo contra uno de los métodos más aplicados por otros colegas.

El resultado es un método más expedito, económico y sencillo porque usa cantidades mínimas de materia prima, tanto de muestra fecal (miligramos, mg) como de la solución etanólica en donde ésta se preservaba (microlitros, $\mu \mathrm{L}$ ), para extraer los nueve $\mathrm{AB}$ utilizados como referencia; asimismo, porque tampoco es necesario usar equipo costoso para la extracción de los AB. Cabe añadir que todos los AB fueron extraídos 
sin importar la materia prima y que también fueron separados de manera más eficiente en los respectivos perfiles de las cuatro especies usadas como referencia, ya que se los pudo distinguir claramente en todas ellas. Además, como resultado de probar el método con las heces de individuos cautivos de los carnívoros silvestres mencionados, también se presentan los perfiles de $\mathrm{AB}$ y Co respectivos y, cabe mencionar, que por primera vez se presenta este tipo de información para el lobo mexicano.

\section{MATERIALES Y MÉTODOS}

Métodos evaluados para la separación cromatográfica en capa fina y visualización de ácidos biliares. Un paso inicial para optimizar el método, fue establecer y adecuar las condiciones básicas necesarias que posteriormente fueron usadas para separar, por cromatografía en capa fina (CCF), los ácidos biliares (AB) y el colesterol (Co) presentes en las heces de los carnívoros silvestres de referencia, conforme a métodos citados en la literatura (Kritchevsky et al. 1963, Roscoe \& Fahrenbach 1963, Siegfried \& Elliott 1968, Chávez \& Krone 1976, Cazón \& Sühring 1999). Para seleccionar el revelador que produjera la mejor visibilidad de las manchas en las cromatoplacas, se usaron los sistemas de fase móvil de Petcoff (Chávez \& Krone 1976) y de Siegfried \& Elliott (1968), evaluándose cada revelador con la correspondiente fase móvil por duplicado. En este paso se seleccionaron ocho reveladores, entre los que se incluyeron tres que aparecen en la literatura y cinco más que nosotros decidimos considerar como sigue: 1. Solución de ácido sulfúrico al 50\% (Cazón \& Sühring 1999). 2. Anisaldehido:ácido acético glacial:ácido sulfúrico concentrado (0.5:50:1 v/v, Kritchevsky et al. 1963). 3. Ácido fosfomolíbdico en solución de etanol al 10\% (Siegfried \& Elliott 1968). 4. p-dimetil-amino-bensaldehído:ácido acético glacial: ácido sulfúrico concentrado (0.5:50:1 w/v/v). 5. vainillina:ácido acético glacial:ácido sulfúrico concentrado $(0.5: 50: 1 \mathrm{w} / \mathrm{v} / \mathrm{v})$. 6. $\beta$-resorcialdehído:ácido acético glacial: ácido sulfúrico concentrado (0.5:50:1 w/v/v). 7. piperonal:ácido acético glacial:ácido sulfúrico concentrado $(0.5: 50: 1 \mathrm{w} / \mathrm{v} / \mathrm{v})$. 8. yodo. Una vez seleccionado el revelador óptimo, se compararon cuatro sistemas de fase móvil (tres mencionados en la literatura y uno propuesto por nosotros), incluyendo: 1. Solución de Petcoff: Hexano:metil-etilcetona:ácido acético (56:36:8 v/v, Chávez \& Krone 1976). 2. Tolueno:ácido acético:agua (5:5:1 v/v, Roscoe \& Fahrenbach 1963). 3. Cloroformo:metanol:ácido acético (80:12:3 v/v, Siegfried \& Elliott 1968). 4. Tolueno:metanol:ácido acético glacial $(60: 20: 3 \mathrm{v} / \mathrm{v})$, los cuales se evaluaron por duplicado respectivamente, usando siempre el revelador de Kritchevsky et al. (1963). Para esta fase inicial, sólo se emplearon Co y AB de referencia (Sigma-Aldrich Chemical ${ }^{\circledR}$ Co., USA) como analitos, considerando entre los últimos a aquellos ácidos que han sido más frecuentemente utilizados en la identificación de carnívoros silvestres, a partir de sus heces como sigue: taurocólico, taurodeoxicólico, glicocólico, cólico, deoxicólico, quenodeoxicólico, ursocólico, litocólico y dehidrocólico. 
Separación cromatográfica en capa fina de los AB y Co de referencia. En todas las evaluaciones cromatográficas de los nueve $\mathrm{AB}$ y Co de referencia, tanto por separado como en conjunto, se aplicaron $1-2 \mu \mathrm{L}$ (en concentración al $0.1 \%$ en solución etanólica) a cromatoplacas de vidrio $(20 \times 20 \mathrm{~cm})$ cubiertas con gel de sílice e indicador para absorción UV a $254 \mathrm{~nm}\left(\right.$ Merck $\left.^{\circledR}\right)$. La cromatoplaca con los estándares y su combinación aplicados se introdujo en un recipiente para CCF, usando la fase móvil a ensayar, de acuerdo a las cuatro opciones mencionadas en la sección anterior. Todas las cromatografías se realizaron bajo condiciones de laboratorio con una humedad ambiental relativa del $28 \pm 2 \%$ y temperatura de $22 \pm 2{ }^{\circ} \mathrm{C}$. Concluida la cromatografía, y previa evaporación de los disolventes orgánicos, la superficie de la placa se roció con el revelador a evaluar (véanse las ocho opciones, conforme a la sección previa); en el caso del yodo, la placa se colocó en un recipiente que contenía gránulos del mismo hasta la visualización de las manchas correspondientes a los estándares de Co y $\mathrm{AB}(\approx 1 \mathrm{hr})$. Las cromatoplacas con un determinado revelador, exceptuando las de yodo, se dejaron por $20-25 \mathrm{~min}$ a $150-160^{\circ} \mathrm{C}$ en un horno (Riossa$\mathrm{HS}^{\circledR}$ ). Las respectivas manchas de los nueve $\mathrm{AB}$ y del Co fueron observadas tanto a simple vista como con una lámpara UV (UVGL-25. UVP, Inc. San Gabriel, CA). Posteriormente, se determinó el Rf (rate of flow o distancia de desplazamiento) de cada mancha (Figs. 2 y 3), el cual es la medida obtenida de dividir el recorrido de los analitos ( $\mathrm{AB}$ y $\mathrm{Co}$ ) desde el punto de aplicación, entre la distancia que se deja desplazar la fase móvil (frente de disolventes) en la cromatoplaca.

Optimización de la separación cromatográfica de AB. Con la finalidad de obtener en una misma cromatoplaca la separación de los nueve $\mathrm{AB}$ utilizados como referencia, se optimizó su Rf (como distancia de separación), utilizando dos sistemas cromatográficos. El primero de ellos se hizo modificando la fase móvil de Siegfried \& Elliott (1968) con la disolución cloroformo:metanol:ácido acético (80:12:0.5 v/v) y una vez que el frente de disolventes alcanzó $18 \mathrm{~cm}$, la cromatoplaca se retiró del recipiente de cromatografía para dejar que se evaporaran los disolventes orgánicos de la fase móvil. En la segunda CCF, se introdujo la misma cromatoplaca en otro recipiente con la misma orientación que en la primera separación cromatográfica, pero esta vez la fase móvil fue de cloroformo:etanol:ácido acético (80:45:3 v/v). En esta ocasión se dejó que el frente de disolventes alcanzara $3 \mathrm{~cm}$ para que cuando se retirara la cromatoplaca y se hubiese evaporado la fase móvil, las señales de los diferentes ácidos biliares fuesen mostradas con el revelador de Kritchevsky et al. (1963). Cabe mencionar que la separación optimizada se replicó siete veces con los AB y el Co de referencia.

Procedencia de las muestras fecales. Se consideraron especies de carnívoros silvestres que ya habían sido abordadas con estos métodos en la literatura, aun cuando los AB todavía presentaran superposición en la separación cromatográfica (v. gr., Capurro et al. 1997, Fernández et al. 1997, Cazón \& Sühring 1999, Guerrero et al. 
2006). Estas especies incluyeron puma (Puma concolor), jaguar (Panthera onca), lobo (la subespecie para México, Canis lupus baileyi, que se examina por primera vez) y coyote (Canis latrans). De cada una de las cuatro especies se analizaron dos muestras fecales de cada uno de dos individuos machos adultos. Todos los animales estaban en condiciones de cautiverio en las instalaciones del Zoológico de Chapultepec, México, D. F., recibiendo la misma dieta, conforme a especificaciones conocidas de nutrición. Las heces frescas de cada uno de los individuos fueron recogidas y depositadas en frascos etiquetados con etanol al 70\% o al 96\%, respectivamente, hasta realizar los ensayos.

Extracción de AB y Co en las heces fecales de los carnívoros de referencia. A diferencia de otros trabajos (Johnson et al. 1984, Quinn \& Jackman 1994, Cazón \& Sühring 1999, Guerrero et al. 2006), esta fase del método se optimizó para realizar los análisis con una cantidad mínima de materia prima, la cual además de incluir las heces húmedas o secas, propiamente, también contempló la solución etanólica en donde las primeras se habían preservado. En esta fase se usaron inicialmente las heces de puma y de jaguar como modelo, ya que al ser de mayor tamaño proveían de más cantidad de materia prima que las heces de los cánidos, pero una vez estandarizado, el procedimiento se repitió con las heces de todas las especies. Para las extracciones se usaron dos sistemas de disolventes orgánicos como mezcla de extracción: diclorometano: metanol (1:1 v/v, D:M sensu Capurro et al. 1997, Fernández et al. 1997, Guerrero et al. 2006) y benceno:metanol (1:1 v/v, B:M sensu Major et al. 1980). Muestras fecales: Ya fuese que se dejaran secar al medio ambiente (heces secas) o que se tomaran directamente del frasco (heces húmedas), se pesaron $50 \mathrm{mg}$ de cada muestra fecal y se transfirieron a tubos Eppendorf a los que se les agregaron $0.5 \mathrm{~mL}$ de la mezcla de extracción (D:M o B:M); se agitaron en vortex (Thermolyne ${ }^{\circledR}$ ) y se centrifugaron a 2000-3000 rpm (Hermle Z233. Labnet ${ }^{\circledR}$ ) durante 1-2 minutos a temperatura ambiente; el sobrenadante fue transferido a un tubo Eppendorf. Solución preservativa de etanol: Se tomó una alícuota de $100 \mu \mathrm{L}$ de la solución de etanol al $70 \%$ o $96 \%$ que sirvió para almacenar las heces y se transfirió a un tubo Eppendorf, permitiendo la evaporación del etanol a temperatura ambiente o en Baño de María; posteriormente, se le agregaron $100 \mu \mathrm{L}$ de D:M o B:M y se agitó. De todas las muestras (heces y solución) con D:M ó B:M se tomaron 1 ó $2 \mu \mathrm{L}$ que se aplicaron a una cromatoplaca; además, esa misma alícuota se tomó directamente de la solución etanólica preservativa y se aplicó directamente sobre la cromatoplaca sin haber pasado por ninguna mezcla de extracción.

Separación por CCF de AB y Co extraídos de las heces fecales. Las CCF para separar los $\mathrm{AB}$ y Co de las heces de las especies de carnívoros de referencia, se desarrollaron considerando: a) la naturaleza de la muestra $(n=4$ heces, secas o húmedas y solución etanólica con y sin mezcla de extracción); b) el medio de extracción (n = 2, D:M y B:M); c) método cromatográfico ( $\mathrm{n}=4$, optimizado con una sola evalua- 
ción cromatográfica, optimizado con dos evaluaciones cromatográficas, método de Siegfried \& Elliott (1968) y método de Petcoff sensu Chávez \& Crone (1976) para compararlo); d) número de muestras por individuo $(\mathrm{n}=2)$; e) número de individuos por especie $(n=2)$ y f) la especie particular ( $n=4$, puma, jaguar, lobo y coyote).

Comparación del método propuesto contra la fase móvil de Petcoff. Los ensayos tanto para elegir el revelador óptimo como para seleccionar las fases móviles y optimizar el Rf con una o dos separaciones cromatográficas, permitieron establecer las condiciones del método propuesto aquí, el cual se comparó contra el método de separación que utiliza la fase móvil de Petcoff, nombrada así por Chávez \& Crone (1976) en honor a Darrell Petcoff, ya que éste es el que más comúnmente se cita en la literatura para identificar carnívoros, a través de la extracción, separación y evaluación de $\mathrm{AB}$ en sus heces, mediante $\mathrm{CCF}$. Estas comparaciones entre el método propuesto, tanto con una o dos separaciones cromatográficas, y la fase móvil de Petcoff se hicieron por triplicado. Cuando todos los aspectos y procedimientos del método fueron optimizados, se efectuaron de manera continua con las heces de especies conocidas de carnívoros. Tomando en cuenta todos los procedimientos y réplicas mencionados en esta y las secciones anteriores que llevaron al nuevo método, el número aproximado de ensayos fue de 100 a 110 cromatoplacas.

\section{RESULTADOS}

Selección de condiciones básicas con ácidos biliares de referencia. Las evaluaciones con cada uno de los ocho reveladores, utilizando los sistemas cromatográficos de Petcoff (Chávez \& Krone 1976) y de Siegfried \& Elliott (1968), permitieron visualizar los 10 analitos de referencia considerados aquí ( 9 ácidos biliares, $\mathrm{AB}$, y el colesterol, Co), tanto a simple vista como con luz UV (Fig. 1). Cabe destacar que la sensibilidad de los ocho reveladores permitió detectar concentraciones de analitos de hasta $10 \mathrm{ng} / \mu \mathrm{L}$. De hecho, algunos reveladores hicieron que las machas adquirieran colores particulares como sucedió con el de Kritchevsky et al. (1963, [p-metoxibenzaldehído (anisaldehído):ácido acético:ácido sulfúrico concentrado, 0.5:50:1 v/v]), la vainillina, el piperonal o el $\beta$-resorcialdehído.

Por otro lado, a partir de realizar seis evaluaciones de separación cromatográfica por cada uno de los cuatro sistemas cromatográficos (fase móvil) utilizados, la fase móvil de cloroformo:metanol:ácido acético (80:12:3 v/v) descrita por Siegfried \& Elliott (1968) resultó tener mejor Rf en la separación de los AB glicocólico, cólico, deoxicólico, quenodeoxicólico, litocólico y dehidrocólico, así como para el Co (Fig. 1A). Con la doble evaluación cromatográfica la separación de los nueve AB y del Co de referencia fue todavía mejor (Fig. 1B), ya que se obtuvo una conspicua diferencia en la separación de todos los $\mathrm{AB}$ evaluados; por ejemplo, con la fase móvil de Pettcof (Fig. 1C) se superponen, por un lado, los AB quenodeoxicólico, dehidrocólico y ur- 


\begin{tabular}{|c|c|c|c|c|c|}
\hline \multicolumn{2}{|c|}{ A } & \multicolumn{2}{|c|}{$\mathbf{B}$} & \multicolumn{2}{|c|}{$\mathrm{C}$} \\
\hline Estándares & $\mathbf{R f}$ & Estándares & $\mathbf{R f}$ & Estándares & $\mathbf{R f}$ \\
\hline Colesterol & 0.85 & Colesterol & 0.85 & Colesterol & 0.83 \\
\hline Dehidrocólico & 0.71 & & & Litocólico & 0.72 \\
\hline Litocólico & 0.68 & & & & \\
\hline \multirow[t]{7}{*}{ Deoxicólico } & 0.65 & & & & \\
\hline & & Dehidrocólico & 0.64 & & \\
\hline & & Litocólico & 0.60 & & \\
\hline & & & & Deoxicólico & 0.58 \\
\hline & & Ursocólico & 0.47 & Quenodeoxicólico & 0.52 \\
\hline & & Quenodeoxicólico & 0.43 & Dehidrocólico & 0.51 \\
\hline & & Deoxicólico & 0.42 & Ursocólico & 0.49 \\
\hline \multirow[t]{3}{*}{ Cólico } & 0.3 & & & & \\
\hline & & Cólico & 0.22 & & \\
\hline & & Glicocólico & 0.13 & Cólico & 0.19 \\
\hline \multirow[t]{5}{*}{ Glicocólico } & 0.08 & & & & \\
\hline & & Taurodeoxicólico & 0.07 & & \\
\hline & & Taurocólico & 0.04 & & \\
\hline & & & & Glicocólico & 0.007 \\
\hline & & & & $\begin{array}{l}\text { Taurodeoxicólico } \\
\text { Taurocólico }\end{array}$ & 0.005 \\
\hline
\end{tabular}

Figura 1. Comparación entre los métodos propuestos en este trabajo (A y B) y el método que se cita más comúnmente en la literatura (C) para separar colesterol Co y ácidos biliares (AB) de referencia (Estándares). A, separación por cromatografía en capa fina (CCF), utilizando el sistema cromatográfico (fase móvil) de Siegfried \& Elliott (1968). B, separación por doble CCF con dos fases móviles distintas. C, separación por CCF usando la solución de Petcoff (sensu Chávez \& Krone 1976). Las manchas de los estándares (•) se revelaron con la solución de Kritchevsky et al. (1963). El nivel de separación de las manchas de los analitos se indica por el valor de su Rf. Ver texto para detalles.

socólico, mientras que por el otro, también se superponen los AB glicocólico y, especialmente, el taurodeoxicólico con el taurocólico. En cambio, con nuestro método de doble separación cromatográfica, sólo se obtiene una ligera superposición entre los AB quenodeoxicólico y deoxicólico (Fig. 1B), pero aun así son distinguibles.

Extracción y separación de ácidos biliares de las muestras fecales de carnívoros. En todos los experimentos $(n=27)$ hubo reproducibilidad y consistencia entre los perfiles de los $\mathrm{AB}$ y Co para las respectivas especies de carnívoros evaluados (Fig. 2), sin importar la naturaleza de la muestra o si se usó la mezcla de extracción. Es decir, que fue posible separar los nueve $\mathrm{AB}$ y Co, tanto de las muestras fecales secas como de las que fueron preservadas en etanol (húmedas), a partir de realizar la extracción con D:M o B:M. Asimismo, también se extrajeron y separaron los AB y el Co de la solución etanólica en que se conservaban las muestras fecales al hacer 


\begin{tabular}{|c|c|c|c|c|c|c|}
\hline $\mathbf{A B}$ & $\mathbf{R f}$ & Estándares & Puma & Jaguar & Lobo mexicano & Coyote \\
\hline & & & $\mathrm{O}$ & $\mathrm{O}$ & $\mathrm{O}$ & \\
\hline \multirow[t]{2}{*}{ Colesterol } & 0.85 & 0 & ○ & - & ○ & 0 \\
\hline & & & O & 0 & & \\
\hline Dehidrocólico & 0.64 & & ○ & $\bullet$ & 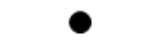 & \\
\hline \multirow[t]{3}{*}{ Litocólico } & 0.60 & ? & & & & \\
\hline & & & 8 & 8 & & \\
\hline & & & 0 & 0 & & O \\
\hline Ursocólico & 0.47 & O & & & & \\
\hline Quenodeoxicolico & 0.43 & & 8 & $\bullet$ & 0 & e \\
\hline \multirow[t]{2}{*}{ Deoxicólico } & 0.42 & & & & & \\
\hline & & & 0 & 0 & & O \\
\hline Cólico & 0.22 & 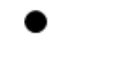 & 8 & 8 & $\bullet$ & $\stackrel{\bullet}{0}$ \\
\hline \multirow[t]{2}{*}{ Glicocólico } & 0.13 & ○ & & $\bullet$ & & \\
\hline & & & & O & & 0 \\
\hline Taurodeoxicólico & 0.07 & & & $\bullet$ & $\bullet$ & \\
\hline Taurocólico & 0.04 & & & & & \\
\hline
\end{tabular}

Figura 2. Comparación del perfil de ácidos biliares (AB) y colesterol (Co) que muestra la eficiencia del método propuesto en muestras fecales de puma (Puma concolor), jaguar (Panthera onca), lobo Mexicano (Canis lupus baileyi) y coyote (Canis latrans), provenientes del Zoológico de Chapultepec, D. F., México. El mismo perfil se obtuvo con doble separación cromatográfica (Fig. 1B), independientemente de la materia prima y del método de extracción. La columna E corresponde a los analitos estándares de los nueve $\mathrm{AB}$ y Co de referencia $(\bullet)$. En las especies se indican tanto las manchas bien visibles $(\bullet)$ de los analitos estándares como la mancha tenue $(\bullet)$ del ácido cólico en el puma, además de ocho analitos desconocidos (०) que también fueron observados. Ver texto para detalles.

la extracción con D:M o B:M. Pero mejor aún, también fue posible obtener el perfil de los $\mathrm{AB}$ y el Co, a partir de evaluar directamente la solución etanólica en que se preservaban las muestras fecales (i. e., sin realizar la previa extracción con D:M o B: M). Además, todos estos perfiles se obtuvieron a partir de $50 \mathrm{mg}$ de muestra fecal, en su caso, y/o de alícuotas de 1 o $2 \mu \mathrm{L}$ del etanol en donde se preservaron.

Descripción del perfil de AB y colesterol en los carnívoros estudiados. En todas las especies de referencia, se detectó ácido cólico y Co (Fig. 2), pero el número y tipo de $A B$ de referencia que fueron revelados varió, de acuerdo con la especie (puma $=5$, jaguar $=6$, lobo mexicano $=5$ y coyote $=3$, Fig. 2). Los dos felinos tuvieron un perfil similar, compartiendo los ácidos dehidrocólico, quenodeoxicólico y 
cólico. Sin embargo, la mancha del ácido cólico fue tenue (círculo gris en la Fig. 2) en el puma, además de que en esta especie se sumó el ácido deoxicólico, mientras que en el jaguar también se detectaron además los ácidos taurodeoxicólico y glicocólico (Fig. 2). Los cánidos compartieron la presencia de los ácidos deoxicólico y cólico, aunque en el lobo mexicano fue exclusiva la presencia de ácido dehidrocólico (presente también en los dos felinos) y del ácido taurodeoxicólico, mientras que en el coyote no se encontró ningún $A B$ exclusivo (Fig. 2). Por otra parte, en las cuatro especies se detectaron manchas con perfiles particulares que no correspondieron con los nueve $\mathrm{AB}$ y el Co usados como referencia en este trabajo, sino a ocho analitos de naturaleza desconocida, los cuales también se detectaron en cierto número de acuerdo con la especie $($ puma $=7$, jaguar $=8$, lobo mexicano $=1$, coyote $=4$ ). Los perfiles de los 17 analitos combinados (9 AB, 8 desconocidos) también contribuyen a identificar diferencias entre las cuatro especies de carnívoros silvestres por sus combinaciones particulares $($ puma $=11$, jaguar $=13$, lobo mexicano $=5$, coyote $=6$, Fig. 2). El jaguar y el coyote muestran mayor cantidad de analitos que el puma y el lobo mexicano, respectivamente, siendo las diferencias en el perfil total de analitos (AB + analitos desconocidos) todavía más evidentes entre los dos cánidos que entre los dos felinos, aún cuando éstos últimos muestran mayor cantidad de analitos totales. Finalmente, cabe destacar que este es el primer trabajo en donde se obtiene el perfil de AB para el lobo mexicano (Canis lupus baileyi) en cautiverio.

\section{DISCUSIÓN}

Características de las muestras y extracción de AB. Todos los aspectos y procedimientos optimizados (Figs. 1 y 2) para obtener un nuevo método para extraer, separar y evaluar AB y Co en heces de carnívoros silvestres, mediante el uso de CCF se resumen en la figura 3. Una aportación notable del método es que se puede realizar utilizando cantidades significativamente menores de materia fecal a las reportadas antes (v. gr., Johnson et al. 1984; Quinn \& Jackman 1994; Cazón \& Sühring 1999; Guerrero et al. 2006), ya que con tan solo $50 \mathrm{mg}$ de muestra fresca o seca, se obtiene un perfil claro y sin alteraciones de los $\mathrm{AB}$. Las ventajas de esto estriban en que la cantidad de muestra fecal utilizada no se convierte en un factor limitante para evaluar el perfil de AB, como lo sugieren Taber et al. (1997) al interpretar los resultados de Watt (1987), además de que lo hace aplicable a otras especies que producen volúmenes fecales mucho menores que los carnívoros analizados ( $v . g r$. , micromamíferos).

En cuanto a la extracción, éste método permite obtener el mismo perfil de $\mathrm{AB}$ y $\mathrm{Co}$ con una variedad de muestras que incluyen hacer extractos a partir de muestras fecales secas, muestras fecales almacenadas en etanol, así como de la misma solución alcohólica en que se preservan las heces, pero mejor todavía, también permite utilizar una alícuota de esa solución, sometiéndola directamente a evaluación cromatográfica (Fig. 3). 


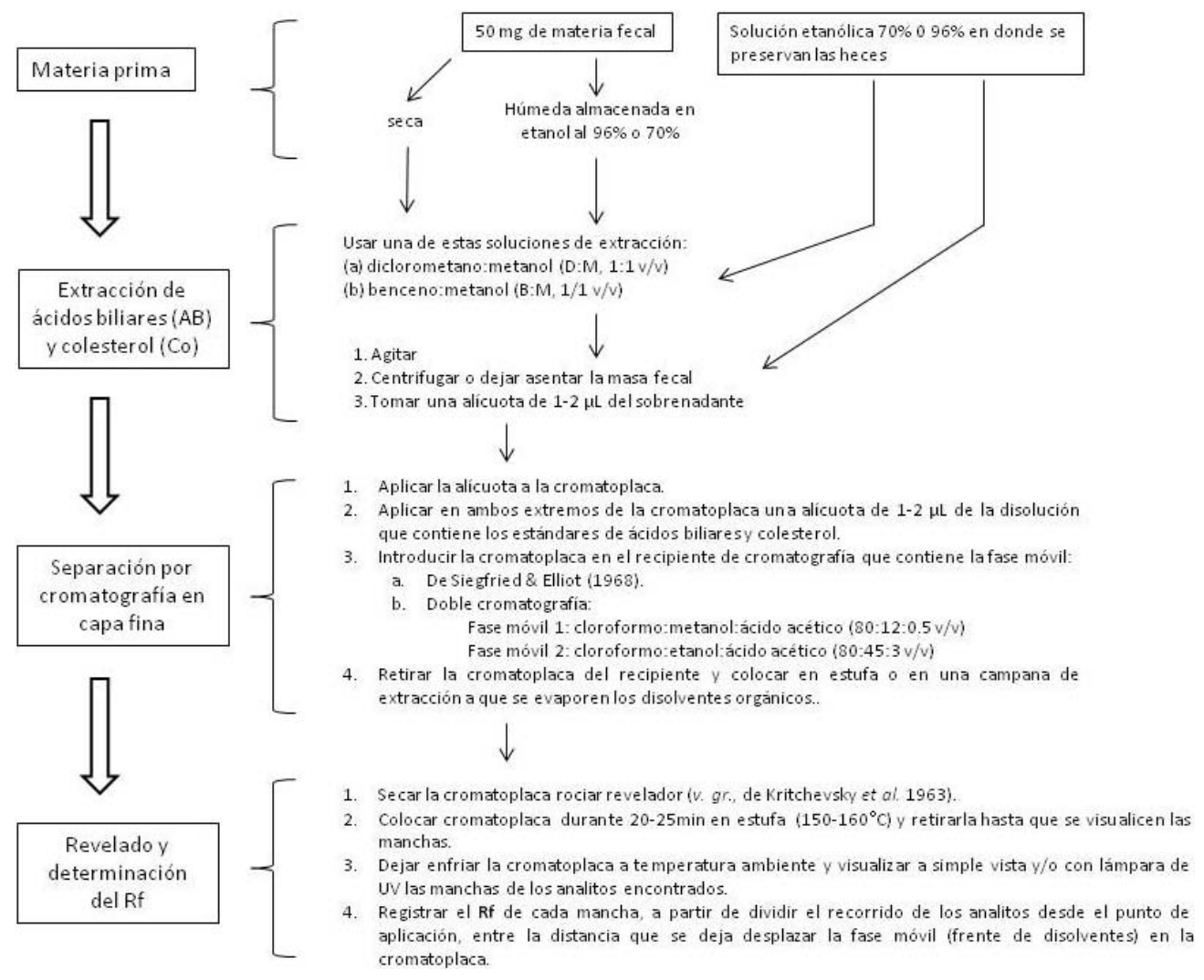

Figura 3. Método optimizado para extraer y evaluar por CCF los AB y Co en cantidades mínimas de heces $(\mathrm{mg})$ de carnívoros silvestres, así como de la solución etanólica $(\mu \mathrm{L})$ en donde se preservaban las mismas.

Esto representa obvias ventajas para poder analizar los AB y Co de muestras fecales que además pueden ser utilizadas para otros fines y que rutinariamente se almacenan en solución etanólica (v. gr., Soto et al. 2004). Una segunda ventaja, es que para realizar la extracción sólo se requieren 15 minutos aproximadamente, en comparación con procedimientos reportados en la literatura (Fernández et al. 1997, Guerrero et al. 2006), los cuales pueden abarcar más de 3 horas.

Condiciones básicas y recomendaciones para la CCF. Por cuanto a los procedimientos para desarrollar la CCF, nuestro método (Fig. 3) optimiza las condiciones básicas del revelador y las fases móviles, además de que mejora el Rf, a partir de dos CCF consecutivas con fases móviles distintas, lo que permite que sea reproducible y que se obtenga una mejor resolución en la separación e identificación de AB, respectivamente (Figs. 1 y 2). En conjunto, estos dos aspectos permitieron separar cinco AB 
(glicocólico, cólico, deoxicólico —o quenodeoxicólico-, litocólico, dehidrocólico) con la fase móvil de Siegfried \& Elliott (1968) si se usa una sola CCF (Fig. 1A), mientras que si se usa la doble CCF con las fases móviles propuestas (Figs. 1B y 3), se pudieron separar los nueve AB examinados (taurocólico, taurodeoxicólico, glicocólico, cólico, deoxicólico, quenodeoxicólico, ursocólico, litocólico, dehidrocólico).

Cabe resaltar que el Rf de los AB taurocólico, taurodeoxicólico y glicocólico, resulta ser muy similar con la fase móvil de Petcoff (Chávez \& Krone 1976) o con la fase móvil de Roscoe \& Fahrenbach (1963), tal y como las utilizaron Guerrero et al. (2006) y Cazón \& Sühring (1999), respectivamente. Esto no permitió separar esos tres $\mathrm{AB}$ adecuadamente y con ello aumentó la incertidumbre en el proceso de identificar a las especies que tuviesen un perfil relacionado con la presencia-ausencia de estos analitos (Fig. 1C). Eso mismo nos sucedió entre los AB deoxicólico y quenodeoxicólico al utilizar las fases móviles de Petcoff (Chávez \& Krone 1976), de Roscoe \& Fahrenbach (sensu Cazón \& Sühring 1999) o la de Siegfried \& Elliott (1968) en una sola CCF.

Una posible solución para distinguir entre las manchas superpuestas de distintos $\mathrm{AB}$, es utilizar un revelador que permita diferenciarlos a partir del color que desarrollan, tal y como sucedió con los reveladores de Kritchevsky et al. (1963), en este y en otros estudios (Khorozyan et al. 2007, Cazón et al. 2009), la vainillina, el piperonal y el $\beta$-resorcialdehído, en este trabajo. Sin embargo, esto no es del todo adecuado y mucho menos se debe considerar que la intensidad del color permita tener una distinción semicuantitativa de los AB, como se ha reportado en la literatura especializada (Major et al. 1980, Quinn \& Jackman 1994, Khorozyan et al. 2007, Cazón et al. 2009), ya que tanto las condiciones específicas del revelador como de las fases móviles pueden variar de laboratorio a laboratorio, haciendo que la distinción entre $A B$ que se superponen sea más bien subjetiva y no esté relacionada con su concentración. Es decir, una aparente derivación lógica podría ser que la alta intensidad del color en la mancha de cierto AB se considerara como indicativa de su mayor concentración; sin embargo, lo anterior también puede deberse, por ejemplo, a una alta concentración del revelador y no del analito. Otra precaución a considerar es que la coloración de cierta mancha puede deberse más bien a la presencia de analitos que eluyen con el mismo Rf que el del AB evaluado y que dependen de la dieta, sexo, edad y salud del individuo en una determinada época del año, aspectos que facilitan cambios en la coloración de esa mancha particular. Por lo tanto, la intensidad y el color de la mancha no necesariamente son características propias del AB bajo análisis y, por ende, no es adecuado utilizarlos como criterios para distinguir entre las especies de interés, hasta conocer qué sustancia(s) o condiciones los hacen diferentes. Lo anterior es importante a considerar, ya que si bien la CCF es un método de separación, también es un procedimiento preparativo para realizar, a posteriori, análisis cuantitativos de los analitos separados. 
En suma, los ensayos realizados nos permiten hacer las siguientes recomendaciones a los investigadores interesados en identificar especies de carnívoros silvestres en vida libre, a partir de los AB en sus heces fecales. Si trabajan tan sólo con los AB: glicocólico; cólico; deoxicólico --o quenodeoxicólico--; litocólico y dehidrocólico, así como con colesterol, la fase móvil de Siegfried \& Elliott (1968) permite una separación (Rf) suficientemente aceptable (Figs. 1A y 3) para determinar perfiles diferenciales entre especies con la metodología de extracción y el revelador de Kritchevsky et al. (1963). Pero si requieren utilizar los nueve AB analizados en este trabajo (taurocólico; taurodeoxicólico; glicocólico; cólico; deoxicólico; quenodeoxicólico; ursocólico; litocólico; dehidrocólico, y colesterol), la metodología de doble CCF que proponemos aquí (Figs. 1B y 3) permite obtener con certidumbre, independientemente del revelador, una separación clara que hace más expedita la identificación no-invasiva de carnívoros. La selección del método, evidentemente dependerá, por ejemplo, del objetivo de cada estudio, de la presencia de diferentes especies de carnívoros que cohabiten en un área determinada y del grado de superposición en sus nichos ecológicos, especialmente en cuanto a sus dietas, pero siempre resultará más certero utilizar un mayor número de analitos como es factible de hacer con el método propuesto.

Perfiles de AB en los carnívoros usados como referencia y la utilidad del método. Los perfiles de los $\mathrm{AB}$ y Co evaluados, tanto de las muestras fecales como de la solución etanólica en que éstas se preservaban, fueron similares a los descritos en la literatura para puma y jaguar (Capurro et al. 1997, Fernández et al. 1997, Taber et al. 1997, Amín et al. 2006, Guerrero et al. 2006), y coyote (Major et al. 1980, Quinn \& Jackman, 1994). Sin embargo, encontramos algunas diferencias, las cuales pueden ser atribuidas al uso de sistemas cromatográficos diferentes o a cómo se utilizó el método en su conjunto, pero también a otras razones. Por ejemplo, el perfil de AB que obtuvimos para coyote fue similar al reportado por Major et al. (1980) y por Quinn \& Jackman (1994), quienes tampoco detectan el ácido quenodeoxicólico, pero en cambio, a diferencia de nosotros, documentan la presencia de ácido litocólico. Esta discrepancia puede deberse a diferencias en el tipo de dieta, como lo arguyen Quinn \& Jackman (1994) ya que la cantidad de fibra vegetal que puede contener el alimento, en combinación o no con el tipo de flora intestinal, influye en el perfil de AB (i. e., documentaron una escasa o nula presencia del ácido quenodeoxicólico en los coyotes alimentados con manzana con respecto a los que tuvieron una dieta a base de alimento para perro Purina ${ }^{\circledR}$ ). Otras causas probables son la "historia” de la dieta alimenticia consumida por los individuos (Quinn \& Jackman 1994, Fernández et al. 1997) y/o las diferentes condiciones climáticas (Khorozyan et al. 2007, Cazón et al. 2009).

A pesar de lo complejo que pareciera el panorama, consideramos que el uso sistemático de una misma metodología, una vez que ha sido simplificada y optimizada 
como aquí, posibilitaría encontrar diferencias en el perfil de $\mathrm{AB}$ en las heces de individuos de una misma especie, o entre diferentes especies, para diversos fines. Esto es importante cuando se involucran varios escenarios y las discrepancias en el perfil de $A B$ que se atribuyen a causas ecofisiológicas, podrían ser más bien el resultado de variaciones metodológicas. Por ello es importante contemplar los referentes ad hoc necesarios, ya que el escenario cambia cuando lo que se quiere es comparar el perfil de $A B$ entre individuos: cautivos o en vida libre; de diferente edad y/o sexo; alojados en diferentes zoológicos y con diferentes dietas; de diferentes especies con la misma dieta; de diferentes especies con dieta desconocida, por mencionar algunos casos. Por ejemplo, si se intenta obtener el perfil especie-específico de AB para cierta especie de carnívoro en cautiverio y se requieren evitar variaciones debidas a factores como la edad, el sexo, el tipo de dieta, resulta conveniente, como se hizo aquí, utilizar animales adultos del mismo sexo y cuya dieta sea conocida para analizar sistemáticamente sus excretas con la misma metodología.

Tal fue el caso para las diferencias entre los perfiles de $A B$ en el lobo mexicano (Canis lupus baileyi) y el coyote (Canis latrans), ya que como ambos se analizaron con machos adultos, mantenidos en cautiverio y con la misma alimentación, el método propuesto permitió constatar diferencias interespecíficas. Además, una comparación del perfil de $\mathrm{AB}$ y Co entre individuos de lobo mexicano (Canis lupus baileyi), alojados en el Zoológico de Chapultepec, México (este trabajo), contra el de animales conespecíficos (Canis lupus spp.) alojados en el Zoológico de Yerevan, Armenia (Khorozyan et al. 2007), arroja que en ambos se presentan manchas correspondientes a los Rf de los ácidos biliares deoxicólico y dehidrocólico, pero también que sus perfiles varían; lo que demuestra la utilidad de esta metodología para distinguir aún a nivel subespecífico, si asumimos que las dietas controladas son semejantes. Con estos dos ejemplos, nuestra posición contrasta con la de Jiménez et al. (1996), quienes al no encontrar diferencias entre las excretas de dos especies de zorra, desalientan el uso de esta metodología para identificar especies congenéricas.

En conclusión, la metodología descrita en este trabajo al ser sencilla, realizable en poco tiempo, fiable y reproducible, permite dilucidar el perfil de ácidos biliares y colesterol, a partir de cantidades mínimas de heces (mg) o de la solución etanólica $(\mu \mathrm{L})$ en donde éstas se preservan, lo que la convierte en una herramienta analítica, indirecta y no invasiva, que puede coadyuvar a la identificación de carnívoros en vida libre a partir de sus heces fecales.

Agradecimientos. Los autores agradecemos a la Dirección General de Zoológicos y Vida Silvestre, su apoyo para la colecta de las muestras fecales en el Zoológico de Chapultepec. Asimismo a dos revisores anónimos por sus sugerencias. Este estudio fue apoyado por la División de Ciencias Biológicas y de la Salud de la UAM-Iztapalapa (proyecto 1440318-ASM y proyecto 1440330-ACC). 


\section{LITERATURA CITADA}

Amín, M., J. C. Faller Menéndez, J. Arroyo Cabrales, C. Chávez, G. Ceballos, R. Núñez, E. Cruz Aldán, M. G. Palacios Mendoza \& A. de la Torre. 2006. Ecología Alimentaria, pp 36-42. In: Chávez, C. \& G. Ceballos (Eds.). Memorias del Primer Simposio. El Jaguar Mexicano en el Siglo XXI: Situación Actual y Manejo. CONABIO-Alianza WWF Telcel-Universidad Nacional Autónoma de México. México D. F.

Capurro A. F., A. J. Novaro, A. Travaini \& M. Romero. 1997. Improved bile acid thin layer chromatography to identify feces of Neotropical carnivores. Journal of Wildlife Management, 61:14241427.

Cazón, A. \& S. S. Sühring. 1999. A technique for extraction and thin layer chromatography visualization of fecal bile acids applied to Neotropical felids scats. Revista de Biología Tropical, 47:245249.

Cazón, A. V., V. D. Juárez, J. A. Monjeau \& M. Lilienfeld. 2009. Discriminación de heces de Puma (Puma concolor) y Jaguar (Panthera onca) por identificación de sus ácidos biliares: Una técnica para el monitoreo de carnívoros silvestres. Mastozoología Neotropical, 16:449-453.

Chávez, M. N. \& C. L. Krone. 1976. Silicic acid thin-layer chromatography of conjugated and free bile acids. Journal of Lipid Research, 17:545-547.

Fernández, G. J., J. C. Corley \& A. F. Capurro. 1997. Identification of cougar and jaguar feces through bile acid chromatography. Journal of Wildlife Management, 61:506-510.

García-Esponda, C. M. G., J. D. Carrera, G. J. Moreira, A. V. Cazón \& L. J. M. De Santis. 2009. Microvertebrados depredados por Leopardus pajeros (Carnivora: Felidae) en el Sur de la Provincia de Mendoza, Argentina. Mastozoología Neotropical, 16:455-457.

Guerrero, C., L. Espinosa, H. M. Niemeyer \& J. A. Simonetti. 2006. Using fecal profiles of bile acids to assess habitat use by threatened carnivores in the Maulino forest of central Chile. Revista Chilena de Historia Natural, 79:89-95.

Haslewood, G. A. D. 1967. Bile salt evolution. Journal of Lipid Research, 8:535-550.

Jiménez, J. E., L. L. Yáñez \& F. M. Jaksic. 1996. Inability of thin-layer chromatography to distinguish feces from congeneric foxes by their bile acid contents. Acta Theriologica, 41:211-215.

Johnson M. K., R. Belden \& D. R. Aldred. 1984. Differentiating Mountain Lion and Bobcat scats. Journal of Wildlife Management, 48:239-244.

Kritchevsky, D., D. S. Martak \& G. H. Rothblat. 1963. Detection of bile acids in thin-layer chromatography. Analitical Biochemistry, 5:388-392.

Khorozyan, I. G., A. Cazon, A. G. Malkhasyan \& A. V. Abramov. 2007. Using thin-layer chromatography of fecal bile acids to study the Leopard (Panthera pardus ciscaucasica) population. Biology Bulletin, 34:361-366.

Major, M., M. K. Johnson, W. S. Davis \& T. F. Kellog. 1980. Identifying scats by recovery of bile acids. Journal of Wildlife Management, 44:290-293.

NOM-059-SEMARNAT. 2010. Protección ambiental -Especies nativas de México de flora y fauna silvestres- Categorías de riesgo y especificaciones para su inclusión, exclusión o cambio- Lista de especies en riesgo. Diario Oficial de la Federación, jueves 30 de diciembre del 2010. México, D. F.

Quinn, T. \& W. R. Jackman. 1994. Influence of diet on detection of fecal bile acids by thin-layer chromatography. Journal of Wildlife Management, 58:295-299.

Ray, J. C. \& M.E. Sunquist. 2001. Trophic relations in a community of African rainforest carnivores. Oecologia, 127:395-408.

Roscoe, H. G. \& M. J. Fahrenbach. 1963. Removal of fecal pigments and its aplication to the determination of fecal bile acids in the rat. Analitical Biochemistry, 6:520-529. 
Siegfried, C. M. \& W. H. Elliott. 1968. Separation of bile acid of rat bile by thin-layer chromatography. Journal of Lipid Research, 9:294-295.

Soto M. A., A. Salame-Méndez, J. Ramírez-Pulido, L. Yanez \& M. A. Armella. 2004. Valoración de hormonas esteroides en heces de una pareja de lobo Mexicano (Canis lupus baileyi) en cautiverio. Acta Zoológica Mexicana (n. s.), 20:187-196.

Taber, A. B., A. J. Novaro, N. Neris \& F. H. Colman. 1997. The food habits of sympatric Jaguar and Puma in the Paraguayan Chaco. Biotropica, 29:204-213.

Watt, E. M. 1987. A scatological analysis of parasites and food habits of jaguar (Panthera onca) in the Cockscomb basin of Belize. M. S. Thesis, University of Toronto, Ontario, Canada. 